\title{
有机场效应晶体管中给体-受体共聚物材料研究进展
}

\author{
夏 昕 $a, c$ 雷 霆 $b, c$ 裴 坚*,b,c 刘晨江*,a,c \\ ( ${ }^{a}$ 石油天然气精细化工教育部＆自治区重点实验室 乌鲁木齐绿色催化与合成技术重点实验室 \\ 新疆大学化学化工学院 乌鲁木齐 830046) \\ ( ${ }^{b}$ 北京大学化学与分子工程学院 北京 100871) \\ ( ${ }^{c}$ 北京分子科学国家实验室 北京 100871)
}

\begin{abstract}
摘要 自 20 世纪 80 年代至今, 有机场效应晶体管(Organic field-effect transistor, OFET)的研究已经取得了很大的发展, 目前可用于场效应晶体管的有机半导体材料已达数百种. 经过 30 年的发展，有机场效应晶体管的迁移率从 $10^{-6} \sim 10^{-5}$ $\mathrm{cm}^{2} \cdot \mathrm{V}^{-1} \cdot \mathrm{s}^{-1}$ 提高到 $12 \mathrm{~cm}^{2} \cdot \mathrm{V}^{-1} \cdot \mathrm{s}^{-1}$, 增长了 6 个数量级, 其性能已经超过了无定形性硅场效应晶体管 $\left(0.1 \sim 1 \mathrm{~cm}^{2} \cdot \mathrm{V}^{-1}\right.$ ・ $\mathrm{s}^{-1}$ ). 值得指出的是，目前该类高性能的材料几乎都是基于给体一受体(Donor-Acceptor，简称 D-A)的共轭聚合物，它也 被誉为第三代半导体材料. 本文综述了近年来国内外 D-A 共聚物半导体材料的研究状况, 对空穴传输型、双极传输型 和电子传输型的 D-A 共聚物半导体材料进行了分类总结和评述，并对其设计思路，器件制备及性能做了详细介绍，总 结了材料的化学结构与器件性能间的基本规律, 为今后应用于有机场效应晶体管的给体-受体共聚物半导体材料提供 设计思路.
\end{abstract}

关键词 有机场效应晶体管; 聚合物材料; 给体-受体共聚物材料

\section{Research Progress in Donor-Acceptor Copolymers Field-Effect Transistors}

\author{
Xia, Xin ${ }^{a, c} \quad$ Lei, Ting ${ }^{b, c} \quad$ Pei, Jian*,b,c Liu, Chenjiang ${ }^{*, a, c}$ \\ ( ${ }^{a}$ Key Laboratory of Oil and Gas Fine Chemicals, Ministry of Education \& Xinjiang Uygur Autonomous Region, Urumqi Key \\ Laboratory of Green Catalysis and Synthesis Technology, College of Chemistry and chemical Engineering, \\ Xinjiang University, Urumqi 830046) \\ ( ${ }^{b}$ College of Chemistry and Molecular Engineering, Peking University, Beijing 100871) \\ ( ${ }^{c}$ Beijing National Laboratory for Molecular Sciences, Beijing 100871)
}

\begin{abstract}
Significant progress has been made in organic field-effect transistors (OFETs) since 1980s. Up to now, hundreds of organic semiconductors used for OFETs have been synthesized. After 30 years of development, the device performance of polymer FETs has been significantly improved, and the mobility has been improved from $10^{-6} \sim 10^{-5} \mathrm{~cm}^{2} \cdot \mathrm{V}^{-1} \cdot \mathrm{s}^{-1}$ to 12 $\mathrm{cm}^{2} \cdot \mathrm{V}^{-1} \cdot \mathrm{s}^{-1}$, surpassing that of amorphous silicon $(\alpha-\mathrm{Si})$ FETs $\left(0.1 \sim 1 \mathrm{~cm}^{2} \cdot \mathrm{V}^{-1} \cdot \mathrm{s}^{-1}\right)$. Note that most of the high-performance polymer FET materials are based on donor-acceptor (D-A) copolymers, which are generally synthesized by the copolymerization between two monomers (donor and acceptor). D-A copolymers have been heralded as the third generation of semiconductor materials, due to their considerable contribution to the fast development of conjugated polymers. In this review, recent studies in D-A copolymer FET materials are summarized from the perspective of design, synthesis, and device performance. Different types of polymer FETs including p-type, ambipolar, and n-type FETs based on D-A copolymers were compared and summarized respectively. It is anticipated to penetrate more deeply the basic relationships among the chemical structure-packing structure-property, which could provide valuable information for the future materials exploitation.
\end{abstract}

Keywords organic field-effect transistor; conjugated polymers materials; donor-acceptor copolymers

\footnotetext{
*E-mail: pxylcj@126.com

Received March 25, 2014; revised April 15, 2014; published online May 6, 2014.

Project supported by the National Natural Science Foundation of China (Nos. 21262035, 21162025), and the Foundation of Beijing National Laboratory for Molecular Sciences.

国家自然科学基金(Nos. 21262035, 21162025)、北京分子科学国家实验室开放课题资助项目.
} 
1977 年, Heeger, MacDiarmid 以及白川英树发现了 高导电率的聚乙炔 ${ }^{[1]}$, 这一发现不仅改变了人们对有机 材料的认识, 还开创了有机电子学这一薮新的领域. 有 机电子学涵盖了有机导体、有机超导体、有机半导体和 有机磁体等相关领域 ${ }^{[2]}$, 其中发展最迅速的是有机半导 体领域. 1986 年, 第一个以聚噻吩为活性层的有机场效 应晶体管(Organic field-effect transistor, OFET) 问世 ${ }^{[3]}$; 同年, $\mathrm{Tang}^{[4]}$ 首次报道了基于唒青和芢衍生物的双层异 质结有机太阳能电池(Organic photovoltaic, OPV), 次年 又报道了第一个基于羟基喹啉衍生物的电致发光二极 管(Organic light-emmiting diode, OLED) ${ }^{[5]}$. 这三类基本 有机电子学器件的成功研制对有机电子学具有里程碑 式的意义. 其中, 有机场效应晶体管是最重要的有机半 导体器件之一, 可以被应用于平板显示器驱动电 路 ${ }^{[6]}$ 、大规模集成电路 ${ }^{[7]}$ 、有机电子标签 ${ }^{[8]}$ 和气体传感 器 [9]等领域.

用于 OFET 的有机半导体材料通常可以分为有机小 分子材料和有机聚合物材料. 由于小分子 OFET 材料具 有纯度高、单晶排列结构易于解析和器件性能好等特点, 目前研究最多的是该类材料. 2011 年, Hasegawa 等 ${ }^{[10]}$ 报 道了通过溶液加工的小分子单晶薄膜器件, 得到了超过 $30 \mathrm{~cm}^{2} \cdot \mathrm{V}^{-1} \cdot \mathrm{s}^{-1}$ 的空穴迁移率. 然而, 高迁移率的小分子 材料要求良好的结晶性, 因此器件加工条件较为苛刻, 另外, 小分子材料无法完全实现柔性器件, 并且其结晶 相态具有热不稳定性. 与小分子材料相比, 聚合物场效 应晶体管材料通常处于无定形性或半结晶状态, 具有更 好的溶液加工特性、更好的机械性能和热力学稳定性, 因此引起了材料学家们的兴趣. 近几年来, 聚合物有机 半导体材料发展十分迅速, 迁移率从 $0.1 \mathrm{~cm}^{2} \cdot \mathrm{V}^{-1} \cdot \mathrm{s}^{-1}$ 提 高到 $10 \mathrm{~cm}^{2} \cdot \mathrm{V}^{-1} \cdot \mathrm{s}^{-1}$ 以上, 增长了近 2 个数量级 ${ }^{[11,12]}$. 发 展高性能的可溶液法加工的新型聚合物有机半导体材 料是目前研究的热点.

导电聚合物材料的发展经历了三个典型的发展阶 段 ${ }^{[13]}$ : 第一代是以聚乙炔为代表的导电聚合物材料, 第 二代是可溶液加工的聚合物有机半导体材料, 而通过给 体片段(donor)和缺电子的受体片段(acceptor)共聚获得 具有窄带隙的共轭高分子材料, 被认为是第三代半导体 聚合物材料. 近两年来, 聚合物场效应晶体管发展十分 迅速, 不仅迁移率有了很大的提高, 空气稳定性问题也 得到了明显的改善, 很多聚合物表现出了超过 1 $\mathrm{cm}^{2} \cdot \mathrm{V}^{-1} \cdot \mathrm{s}^{-1}$ 的迁移率. 给体-受体(Donor-Acceptor, 简称 D-A)共聚物作为第三代半导体聚合物在场效应晶体管 中显示出了较理想的器件性能, 取得了较好的成果, 其 中体异质结太阳能电池的效率超过 $9 \%^{[14]}$, 而场效应晶 体管材料的空穴迁移率则达到了 $12 \mathrm{~cm}^{2} \cdot \mathrm{V}^{-1} \cdot \mathrm{s}^{-1}$. 这些
迁移率大于 $1 \mathrm{~cm}^{2} \cdot \mathrm{V}^{-1} \cdot \mathrm{s}^{-1}$ 的高性能 D-A 共聚物大都是 经过钯催化的偶联反应(例如, Stille 偶联反应, SuzukiMiyaura 偶联反应, Sonogashira 偶联反应, Heck 偶联反 应等 ${ }^{[15 ~ 17]}$ 得到的. D-A 共聚物的设计过程中, 可选择的 富电子给体片段较为丰富, 而缺电子的受体片段则较 少, 缺电子片段的设计也是 D-A 共聚物的研究核心之 一. 近几年, 一系列基于苯并噻二唑(BT)、并吡咯二酮 (DPP)、苯并二噻二唑(BBT)、嵴二酰亚胺(NDI)和异靛 青(II 或 IID)等缺电子受体片段被用于聚合物场效应晶 体管材料中, 很多聚合物表现出了超过 $1 \mathrm{~cm}^{2} \cdot \mathrm{V}^{-1} \cdot \mathrm{s}^{-1}$ 的 迁移率. 图 1 给出了这几种常用的缺电子受体片段的化 学结构和其最高占有轨道(HOMO)/最低未占有轨道 (LUMO)的能级 ${ }^{[18]}$. 从图中可以看出, 这些化学结构的 $\mathrm{HOMO}$ 能级大概在 $-5.3 \mathrm{eV}$ 到 $-6.7 \mathrm{eV}$ 之间, 而 LUMO 能级则明显较低, 位于 $-3.4 \mathrm{eV}$ 到 $-3.8 \mathrm{eV}$. 从结构上 看, 通过引入酮羰基和噻二唑等强吸电子官能团, 受体 片段的 LUMO 能级可以得到有效的降低, 如 DPP, BT, BBT.

基于这一领域的快速发展，本文系统总结了近几年 D-A 共聚物在有机场效应晶体管中的研究进展, 介绍了 有机场效应晶体管的类型及结构对有机场效应晶体管 性能的影响, 并对空穴传输型、双极传输型和电子传输 型的给体-受体聚合物半导体材料进行了分类总结和评 述.

\section{1 有机场效应晶体管的基本器件类型及结构}

如前所述, D-A 共聚物的分子结构对于聚合物场效 应晶体管的性能有着至关重要的作用. 然而, 器件的结 构对于场效应晶体管性能的影响也是不容忽视的.

有机场效应晶体管是由有机半导体活性层 (Semiconductor)、栅极绝缘层(Insulator) 以及源极 (Source)、漏极(Drain)和栅极(Gate)等三个电极组成的器 件. 其中源、漏两极分别与半导体材料直接接触，而㮽 极则由绝缘层将其与半导体隔开. 向沟道流入载流子的 电极称为源极, 由沟道流出载流子的电极称为漏极. 在 栅极加上偏压的情况下，电场能够诱导半导体层中产生 电荷，并在源漏两极之间产生电流. 场效应晶体管中根 据多数载流子的类型可以分为 $\mathrm{p}$ 型(空穴)场效应晶体管 (p-type FET) 和 $n$ 型(电子)场效应晶体管(n-type FET), 既 可传输电子又可传输空穴的场效应晶体管称为双极传 输型场效应晶体管(Ambipolar FET). 如图 2 所示, OFET 的器件构型包括: (a)底柾顶接触(BGTC), (b) 底栅底接触 (BGBC), ( c) 顶栅顶接触 (TGTC) 以及 (d) 顶柱底接触 (TGBC) 四种. 这四种器件结构的区别在不同的栅极和 源漏极位置和加工顺序上. 通常, 加工 $\mathrm{p}$-型场效应晶体 


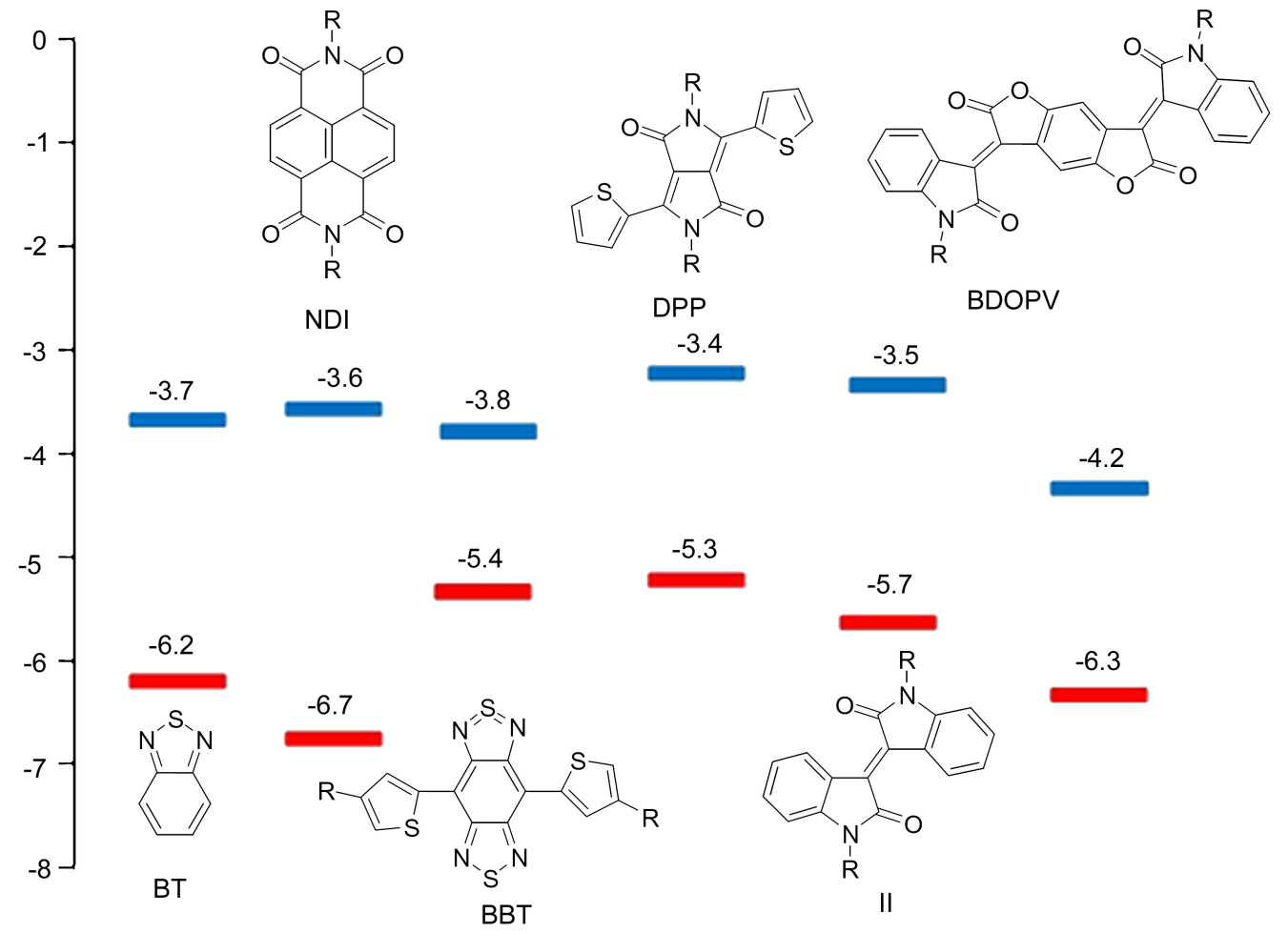

图 1 几种重要的缺电子受体片段和其 $\mathrm{HOMO} / \mathrm{LUMO}$ 能级 $(\mathrm{eV})$

Figure 1 HOMO and LUMO levels for the acceptors described in this perspective $(\mathrm{eV})$
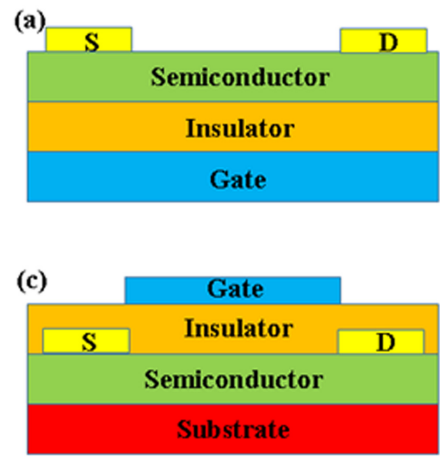

(b)
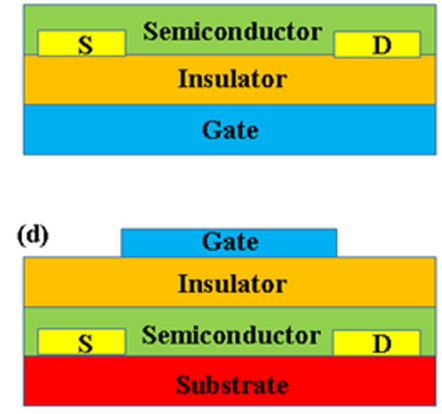

图 2 常见的 OFET 器件剖面结构示意图

Figure 2 Transistor structures described in this perspective

(a) The bottom-contact bottom-gate structure, (b) the top-contact bottom-gate structure, (c) the bottom-contact top-gate structure, and (d) the top-contact top-gate structure

管时采用底柾顶接触结构; 而在加工双极传输型和 $n$-型 场效应晶体管时, 顶栅底接触结构的器件则表现出更好 的性能.

有机场效应晶体管的性能通常由三个主要的参数 来衡量: 场效应迁移率 $(\mu)$, 电流开关比 $\left(I_{\mathrm{on}} / I_{\mathrm{off}}\right)$ 以及阈值 电压 $\left(V_{\mathrm{th}}\right)$. 三个性能参数均可以从场效应晶体管的转移 特性曲线 ( $I_{\mathrm{SD}}$ 对 $V_{\mathrm{G}}$ 作图)、输出特性曲线 ( $I_{\mathrm{SD}}$ 对 $V_{\mathrm{SD}}$ 作图) 获得. 在一定的源漏电压 $\left(V_{\mathrm{SD}}\right)$ 下, 改变栅极电压 $\left(V_{\mathrm{G}}\right)$ 和 测试源漏电流 $\left(I_{\mathrm{SD}}\right)$ 可以获得转移特性曲线. 在一定的栅 极电压 $\left(V_{\mathrm{G}}\right)$ 下, 改变源漏电压 $\left(V_{\mathrm{SD}}\right)$ 和测试源漏电流 $\left(I_{\mathrm{SD}}\right)$
可以获得输出特性曲线(图 3).

当源漏电压较小 $\left(V_{\mathrm{SD}}<\left|V_{\mathrm{G}}-V_{\mathrm{th}}\right|\right)$ 时, 电荷密度从源 极到漏极线性减小, 此时源漏电极之间的电流大小正比 于源漏电压的大小, 有机场效应晶体管工作在线性区, 线性区电流的大小为:

$$
I_{\mathrm{DS}}=\frac{W}{L} \mu_{\mathrm{lin}} C_{\mathrm{i}}\left(V_{\mathrm{G}}-V_{\mathrm{T}}\right) V_{\mathrm{DS}}
$$

当漏电压增大至接近于栅压时 $\left(V_{\mathrm{SD}}=\left|V_{\mathrm{G}}-V_{\mathrm{th}}\right|\right)$, 漏 极处的电势减小至零, 进一步增大源漏电压, 此时源漏 电流就不再依赖于漏极偏压, 源漏电极之间的电流达到 

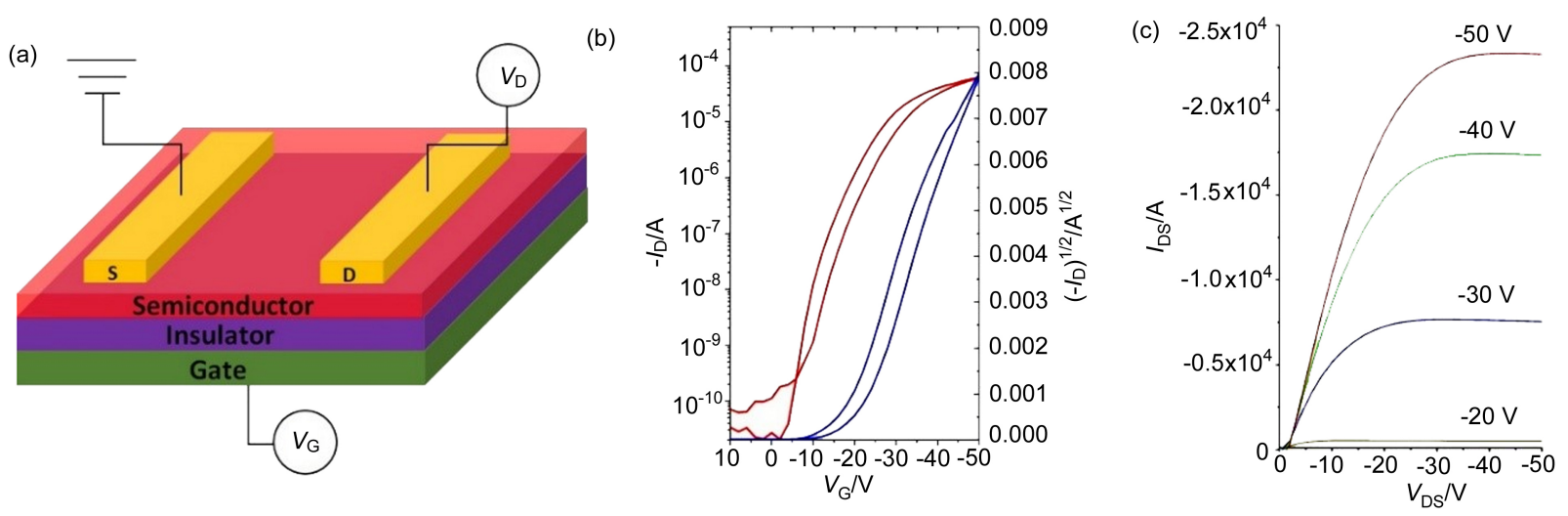

图 3 (a)常见的有机场效应晶体管的器件结构; 场效应管的(b)转移特性曲线和(c)输出特性曲线

Figure 3 (a) Device structure common organic field-effect transistors, (b) transfer characteristic curve, and (c) output characteristic curve

饱和, 有机场效应晶体管工作在饱和区, 饱和区电流的 大小为:

$$
I_{\mathrm{DS}}=\frac{W}{2 L} \mu_{\mathrm{sat}} C_{\mathrm{i}}\left(V_{\mathrm{G}}-V_{\mathrm{T}}\right)^{2}
$$

其中 $W$ 是沟道宽度, $L$ 是沟道长度, $C_{\mathrm{i}}$ 是绝缘层单位面 积的电容.

\section{$2 \mathrm{p}$ 型共轭高分子材料}

传统上用于场效应晶体管研究的 $\mathrm{p}$ 型聚合物材料主 要是噻吩类聚合物, 其中最为成功的例子便是聚 (3-己 基噻吩)(P3HT)体系. 但是该类聚合物迁移率较低并且 在空气中稳定性较差, 很难满足进一步的商业化要求. 而近几年, 越来越多的 $\mathrm{p}$ 型 D-A 共聚物材料 OFET 器件 表现出了大于 $1 \mathrm{~cm}^{2} \cdot \mathrm{V}^{-1} \cdot \mathrm{s}^{-1}$ 的空穴迁移率, 并且显示了 良好的空气稳定性和热稳定性.

2010 年, McCulloch 等 ${ }^{[19]}$ 分别通过 Suzuki 和 Stille 反应合成了基于苯并噻二唑的 D-A 共聚物 IDT-BT (1) 和聚合物 IDT-TT (2) (图 4) (表 1, 化合物 1 和 2), 这两 个化合物表现出良好的场效应性能. 其中, IDT-BT 在薄 膜下采取 face-on 的排列模式, $\pi-\pi$ 堆积距离为 $4.1 \AA$, 采 用 TGBC 器件结构最高空穴迁移率达到了 $1.2 \mathrm{~cm}^{2} \cdot \mathrm{V}^{-1}$. $\mathrm{s}^{-1}$. 而 IDT-TT 器件的空穴迁移率仅为 $0.15 \mathrm{~cm}^{2} \cdot \mathrm{V}^{-1}$. $\mathrm{s}^{-1}$, 这主要是因为其薄膜的结晶性较差. 由于 IDT-BT 展示了良好的场效应性能, 预计基于苯并噻二唑的 D-A 共聚物将被广泛应用于有机电子学领域. 2011 年, Müllen 等 ${ }^{[20]}$ 报道了以苯并噻二唑为受体, 环戊二烯并 二塞吩为给体的 D-A 共聚物 BTZ-CDT-C16 (3) (图 4) (表 1, 化合物 3), 通过提高聚合物的分子量, 其空穴迁 移率达到 $3.3 \mathrm{~cm}^{2} \cdot \mathrm{V}^{-1} \cdot \mathrm{s}^{-1}$. 在此基础上, 他们通过溶液 加工该聚合物的纳米线场效应晶体管，测得了 5.5 $\mathrm{cm}^{2} \cdot \mathrm{V}^{-1} \cdot \mathrm{s}^{-1}$ 的空穴迁移率 ${ }^{[21]}$. 这一系列研究证明, 聚合 物的分子量和分子间排列对迁移率都有着至关重要的
影响.

吡咯并吡咯二酮(DPP)是一种新型杂环红色染料, 它具有着色能力高, 分散性良好, 遮盖性优异, 耐酸碱 等优点. 1974 年, Siegal 等 ${ }^{[22]}$ 在试图制备内酰胺类化合 物时，首次通过 Reformasky 反应合成了苯基取代的 DPP 结构, 但是产率较低. 而 Ciba 公司 ${ }^{[23]}$ 成功开发了以 丁二酸酯和苯腈为原料的合成路线，步骤简便，原料易 于得到，被广泛应用. DPP 作为染料时多是基于苯的衍 生物，但是，由于苯基较大的空间位阻作用，苯基取代 的 DPP 不适合用于有机电子学材料. Zhu 等 ${ }^{[24]}$ 以氧基噻 吩取代苯腈为原料, 合成了噻吩取代的 DPP 的单体. 随 后，呋喃、硒吩、并二噻吩等取代的 DPP 随之发展起来 并且被应用于聚合物场效应晶体管和太阳能电池材料 之中.

2011 年, Dodabalapur 等 ${ }^{[25,26]}$ 合成了以噻吩取代的 DPP 为受体, 以荎为给体的 D-A 共聚物 PDPP-TNT (4) (图 4) (表 1, 化合物 4), 显示良好的场效应性能, 其最大 空穴迁移率接近 $1 \mathrm{~cm}^{2} \cdot \mathrm{V}^{-1} \cdot \mathrm{s}^{-1}$. 其聚合物薄膜在 $140{ }^{\circ} \mathrm{C}$ 下热退火处理, $X$ 射线衍射和原子力显微镜(AFM)的结 果表明，该聚合物结晶度有了显著改善. Toney 等 ${ }^{[27]}$ 以 噻吩取代的 DPP 为受体, 联二噻吩为给体合成了 D-A 共聚物 DPPT-2T (5) (图 4) (表 1, 化合物 5), 其最大的空 穴迁移率为 $0.74 \mathrm{~cm}^{2} \cdot \mathrm{V}^{-1} \cdot \mathrm{s}^{-1}$. Choi 等 ${ }^{[28]}$ 使用相同的增溶 基团(2-辛基十二烷), 得到了分子量约为 $60 \mathrm{~kg} / \mathrm{mol}$ 的 D-A 共聚物, 其空穴迁移率提高到 $1.04 \mathrm{~cm}^{2} \cdot \mathrm{V}^{-1} \cdot \mathrm{s}^{-1}$. 随 后，他们使用更为富电子的联二硒吩作为给体合成了聚 合物 P(DPP-alt-DTBSe) (6) (图 4) (表 1，化合物 6)，其空 穴迁移率达到 $1.5 \mathrm{~cm}^{2} \cdot \mathrm{V}^{-1} \cdot \mathrm{s}^{-1}$. X 射线衍射结果表明, P(DPP-alt-DTBSe) 比 DPPT-2T 具有更好的结晶度, 对于 同样的增溶基团，其面面间距(18.5 ̊)比 DPPT-2T (18.9 $\AA)$ 更短. 同时, $\mathrm{Li}$ 等 ${ }^{[29]}$ 合成了相同的 D-A共聚物 5, 该聚 合物在不进行退火的情况下空穴迁移率就达到 0.89 


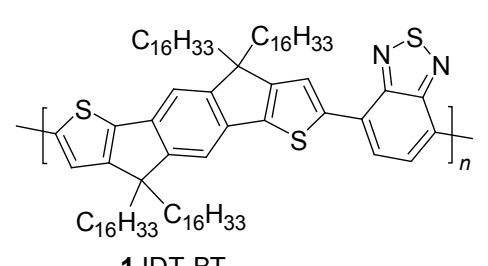

1 IDT-BT

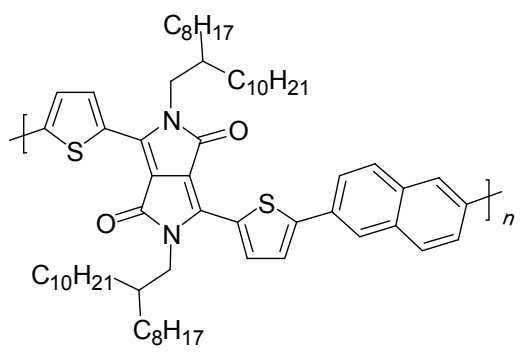

4 PDPP-TNT

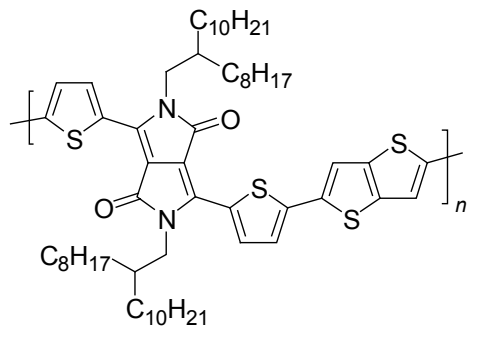

7 PDBT-co-TT

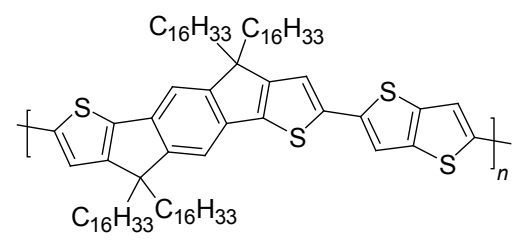

2 IDT-TT

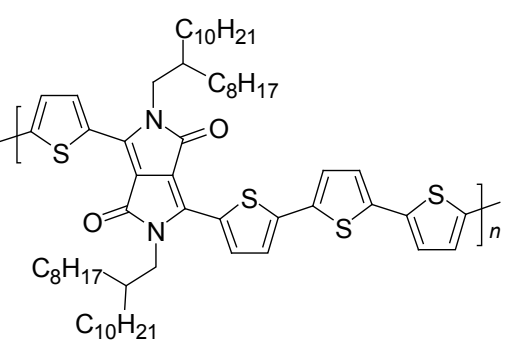

5 DPPT-2T

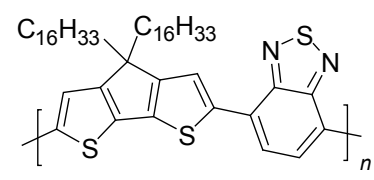

3 CDT-BTZ-C16

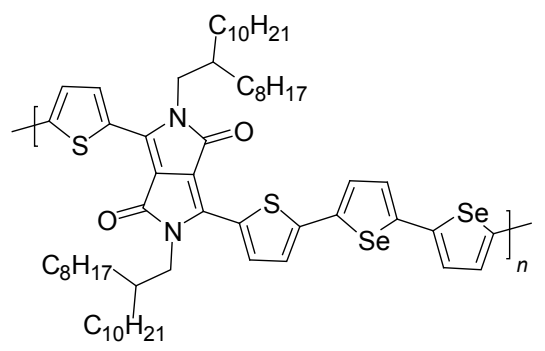

6 P(DPP-alt-DTBSe)

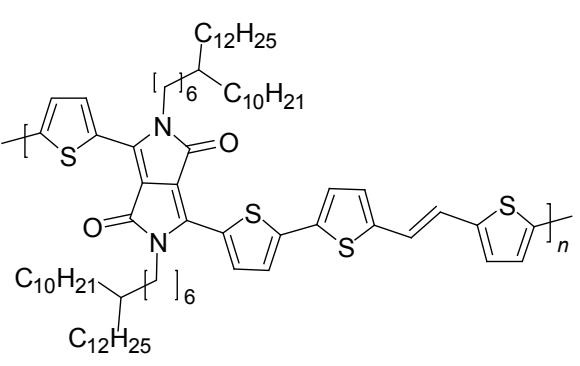

8a P-29-DPPDBTE

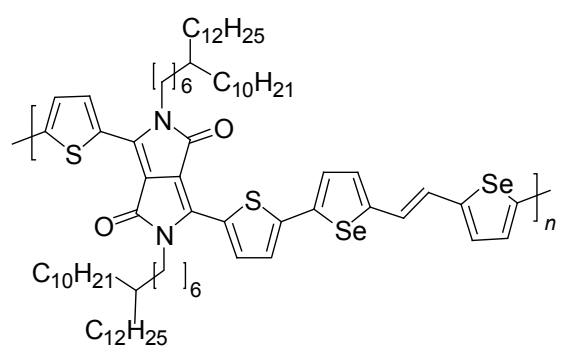

8b P-29-DPPDTSE

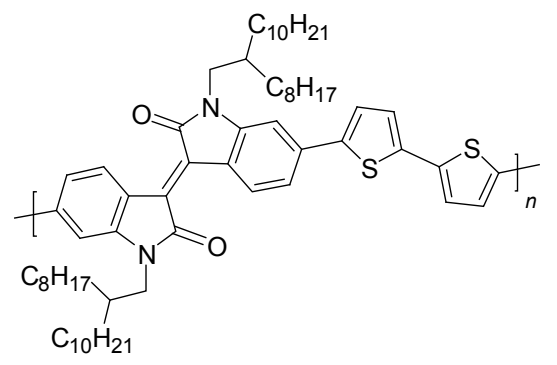

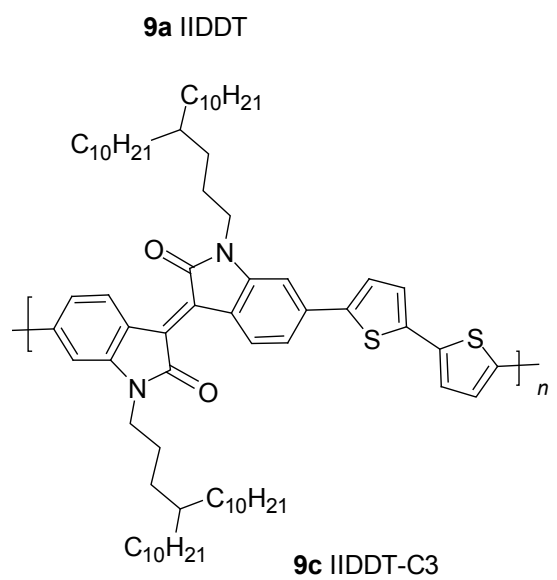
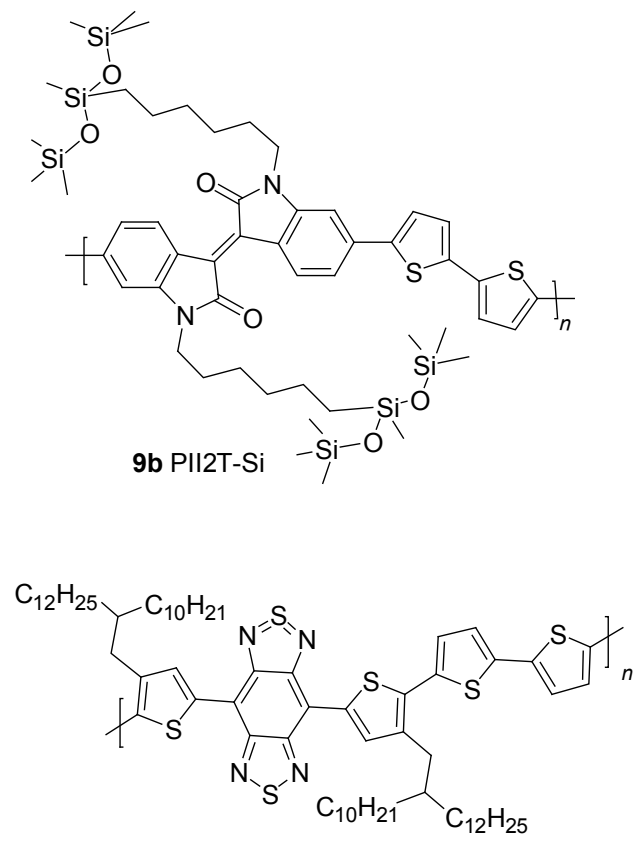

10 PBBTQT

图 $4 \mathrm{p}$ 型聚合物场效应材料的分子结构 $\mathbf{1} \sim \mathbf{1 0}$

Figure 4 Molecular structures of p-type host materials $\mathbf{1} \sim \mathbf{1 0}$ 
表 1 D-A 共聚物场效应材料的载流子迁移率、HOMO/LUMO 能级以及器件性能

Table 1 Electronic properties and OFET device data for polymers based on D-A copolymer

\begin{tabular}{|c|c|c|c|c|c|c|c|c|}
\hline D-A copolymer & $\begin{array}{c}M_{\mathrm{n}} / M_{\mathrm{w}} \\
(\mathrm{kg} / \mathrm{mol})\end{array}$ & $\begin{array}{c}\mathrm{HOMO} / \\
\mathrm{eV}\end{array}$ & $\begin{array}{c}\text { LUMO/ } \\
\mathrm{eV}\end{array}$ & $\begin{array}{c}\mu_{\text {hole }} / \\
\left(\mathrm{cm}^{2} \cdot \mathrm{V}^{-1} \cdot \mathrm{s}^{-1}\right)\end{array}$ & $\begin{array}{c}\mu_{\text {electron }} / \\
\left(\mathrm{cm}^{2} \cdot \mathrm{V}^{-1} \cdot \mathrm{s}^{-1}\right)\end{array}$ & $I_{\text {on }} / I_{\text {off }}$ & Device structure & Ref. \\
\hline 1 IDT-BT & $38 / 108$ & -5.4 & -4.7 & 1.25 & $\mathrm{~N} / \mathrm{A}$ & $10^{3} \sim 10^{4}$ & TGBC, tested in $\mathrm{N}_{2}$ & [19] \\
\hline 2 IDT-TT & $64 / 168$ & -5.4 & -4.6 & 0.17 & $\mathrm{~N} / \mathrm{A}$ & $10^{6}$ & TGBC, tested in $\mathrm{N}_{2}$ & [19] \\
\hline 3 CDT-BTZ-C16 & $51 /-$ & -5.3 & -3.6 & 5.5 & $\mathrm{~N} / \mathrm{A}$ & $10^{5} \sim 10^{6}$ & BGTC, tested in $\mathrm{N}_{2}$ & {$[20,21]$} \\
\hline 4 PDBT-co-NAP & $64 / 91$ & -5.29 & -3.79 & 0.98 & $\mathrm{~N} / \mathrm{A}$ & $10^{7}$ & TGBC, tested in $\mathrm{N}_{2}$ & {$[25,26]$} \\
\hline 5 DРPT-2T & $90 /-$ & -5.2 & -4 & 1.04 & N/A & $10^{5} \sim 10^{6}$ & TGBC, tested in $\mathrm{N}_{2}$ & [27] \\
\hline 6 P(DPP-alt-DTBSe) & $19 /-$ & N/A & N/A & 1.5 & $\mathrm{~N} / \mathrm{A}$ & $10^{6}$ & BGTC, tested in $\mathrm{N}_{2}$ & {$[28]$} \\
\hline 7 PDBT-co-TT & $110 / 501$ & -5.25 & -3.4 & 10.5 & N/A & $10^{6} \sim 10^{8.5}$ & BGTC, tested in $\mathrm{N}_{2}$ & {$[10]$} \\
\hline 8a P-29-DPPDBTE & $36 / 58$ & -5.25 & N/A & 10.54 & N/A & $10^{6}$ & BGTC, tested in $\mathrm{N}_{2}$ & [31] \\
\hline 8b P-29-DPPDTSE & $33 / 60$ & -5.27 & N/A & 12.04 & $\mathrm{~N} / \mathrm{A}$ & $10^{6}$ & BGTC, tested in $\mathrm{N}_{2}$ & {$[31]$} \\
\hline 9a IIDDT & $33.7 / 181.98$ & -5.7 & -3.7 & 0.79 & $\mathrm{~N} / \mathrm{A}$ & $10^{7}$ & BGTC, tested in $\mathrm{N}_{2}$ & {$[33]$} \\
\hline 9b PII2T-Si & $138 / 454$ & -5.2 & -3.58 & 2.5 & $\mathrm{~N} / \mathrm{A}$ & $10^{6}$ & BGTC, tested in $\mathrm{N}_{2}$ & {$[35]$} \\
\hline 9c IIDDT-C3 & $39.2 / 125.44$ & -5.52 & -3.74 & 3.62 & $\mathrm{~N} / \mathrm{A}$ & $10^{6}$ & BGTC, tested in $\mathrm{N}_{2}$ & {$[36]$} \\
\hline 10 PBBTQT & $65.9 /-$ & -4.6 & -3.8 & 2.5 & N/A & N/A & BGBC, tested in $\mathrm{N}_{2}$ & {$[37]$} \\
\hline 11 PDPP3T & $54 /-$ & -5.3 & -4 & 1.57 & 0.18 & $\mathrm{~N} / \mathrm{A}$ & BGTC, tested in Vacuum & {$[41,42]$} \\
\hline 12 HD-PPTV & $56 / 154$ & -5.14 & -3.34 & 0.17 & 0.019 & $10^{2} \sim 10^{3}$ & BGBC, tested in $\mathrm{N}_{2}$ & {$[43]$} \\
\hline 13a РBBT6DPP & $8.7 /-$ & -4.55 & -3.9 & 0.83 & 1.36 & $\mathrm{~N} / \mathrm{A}$ & BGBC, tested in $\mathrm{N}_{2}$ & {$[44]$} \\
\hline 13b PBBT12DPP & $8.8 /-$ & -4.55 & -4.55 & 1.17 & 1.32 & $\mathrm{~N} / \mathrm{A}$ & $\mathrm{BGBC}$, tested in $\mathrm{N}_{2}$ & {$[44]$} \\
\hline 14 PSeDPP & $96.6 / 204.8$ & -5.09 & -3.46 & 1.62 & 0.14 & $10^{4}$ & BGTC, tested in $\mathrm{N}_{2}$ & {$[45]$} \\
\hline 15 PTDPPSe-Si & $23.3 / 70.4$ & -5.10 & -3.49 & 8.84 & 4.34 & $10^{4}$ & BGTC, tested in $\mathrm{N}_{2}$ & {$[46,47]$} \\
\hline 16 PFII2T & $75.7 / 195.3$ & -5.46 & -3.86 & 1.85 & 0.51 & $10^{5} \sim 10^{6}$ & TGBC, tested in air & [48] \\
\hline 17 PCII2T & $60 / 126$ & -5.64 & -3.86 & 0.54 & 0.48 & $10^{4} \sim 10^{5}$ & TGBC, tested in air & {$[49]$} \\
\hline $18 \mathrm{PCII} 2 \mathrm{Se}$ & $60 / 126$ & -5.57 & -3.84 & 0.81 & 0.66 & $10^{5} \sim 10^{6}$ & TGBC, tested in air & [49] \\
\hline 19 P(PDI2OD-DD) & $10 / 15$ & -5.9 & -3.9 & N/A & 0.013 & $10^{4}$ & BGTC, tested in $\mathrm{N}_{2}$ & {$[51]$} \\
\hline 20 P(NDIO2D-T2) & $52.5 / 288.9$ & -5.61 & -3.96 & $\mathrm{~N} / \mathrm{A}$ & 0.85 & $10^{6} \sim 10^{7}$ & BGTC, tested in Vacuum & {$[52]$} \\
\hline 21 N-CS2DPP-OD-TEG & $67.3 / 314$ & -5.38 & -3.66 & $\mathrm{~N} / \mathrm{A}$ & 3.00 & $\mathrm{~N} / \mathrm{A}$ & TGBC, tested in $\mathrm{N}_{2}$ & {$[54]$} \\
\hline 22 DPPPhF4 & $16.3 / 29.2$ & -5.65 & -4.18 & N/A & 2.36 & N/A & BGTC, tested in $\mathrm{N}_{2}$ & {$[55]$} \\
\hline $23 \mathrm{BDPPV}$ & $37.6 / 89.49$ & -6.12 & -4.1 & $\mathrm{~N} / \mathrm{A}$ & 1.1 & N/A & TGBC, tested in air & {$[60]$} \\
\hline 24 BDOPV-2T & $77.2 / 231.6$ & -5.72 & -4.15 & N/A & 1.74 & $\mathrm{~N} / \mathrm{A}$ & TGBC, tested in air & {$[61]$} \\
\hline 25 FBDPPV-1 & $66.3 / 128.9$ & -6.19 & -4.26 & N/A & 1.70 & $10^{5} \sim 10^{6}$ & TGBC, tested in air & {$[62]$} \\
\hline 26 FBDPPV-2 & $53.8 / 164.7$ & -6.22 & -4.30 & N/A & 0.81 & $10^{4} \sim 10^{5}$ & TGBC, tested in air & {$[62]$} \\
\hline
\end{tabular}

$\mathrm{cm}^{2} \cdot \mathrm{V}^{-1} \cdot \mathrm{s}^{-1}, 100{ }^{\circ} \mathrm{C}$ 退火后迁移率进一步提高到 0.97 $\mathrm{cm}^{2} \cdot \mathrm{V}^{-1} \cdot \mathrm{s}^{-1}$. Ha 和 Zhang 所测得的空穴迁移率都是在 $150{ }^{\circ} \mathrm{C}$ 下热退火处理其聚合物薄膜得到的. 这个结果表 明, 较低的退火温度对于在柔性衬底上进行器件加工是 非常有意义的. 2010 年, Sonar 等 ${ }^{[12]}$ 首次报道了以并二噻 吩作为给体的聚合物 PDBT-co-TT (7) (图 4) (表 1, 化合 物 7), 其空穴迁移率为 $0.94 \mathrm{~cm}^{2} \cdot \mathrm{V}^{-1} \cdot \mathrm{s}^{-1} .2012$ 年, Ong 等 ${ }^{[11]}$ 使用同样的聚合物, 大幅提高 PDBT-co-TT 聚合物 的分子量并使用 $\mathrm{BGBC}$ 器件结构后空穴迁移率迅速提 高到 $10.5 \mathrm{~cm}^{2} \cdot \mathrm{V}^{-1} \cdot \mathrm{s}^{-1} .2012$ 年, $\mathrm{Liu}$ 等 $^{[30]}$ 首先合成以噻 吩乙烯噻吩为给体片段的聚合物 PDVT-8 和 PDVT-10, 当引入的烷基链不同时，其空穴迁移率达到了 8.2 $\mathrm{cm}^{2} \cdot \mathrm{V}^{-1} \cdot \mathrm{s}^{-1} .2013$ 年, $\mathrm{Kim}$ 等 ${ }^{[31]}$ 同样合成了以噻吩乙烯
噻吩为给体片段的聚合物 P-29-DPPDBTE (8a), 同时, 合成了以硒吩乙烯硒吩 P-29-DPPDTST (8b) (图 4) (表 1, 化合物 $8 \mathrm{a}$ 和 $8 \mathrm{~b})$, 两个聚合物显示了极其良好的场效应 性能，P-29-DPPDBTE (8a)使用 7-癸基十二烷作为增溶 基团, 空穴迁移率为 $10.54 \mathrm{~cm}^{2} \cdot \mathrm{V}^{-1} \cdot \mathrm{s}^{-1}, \mathrm{P}-29-\mathrm{DPPDTST}$ (8b) 使用了同样的增溶基团，其空穴迁移率高达到 $12.04 \mathrm{~cm}^{2} \cdot \mathrm{V}^{-1} \cdot \mathrm{s}^{-1}$, 是至今为止空穴迁移率的最高值.

靛青(Indigo)是一类非常著名的染料，广泛用于印 染行业. 而作为其异构体的异靛青(Isoindigo)用于染料 研究的却不多. 受到并吡咯二酮(DPP)类染料在太阳能 电池中应用的启发, Reynolds 等 ${ }^{[32]}$ 利用异靛青设计并合 成了一系列基于异靛青的 D-A 共聚物，表现出了较窄的 能隙, 并应用于太阳能电池之中. 我们研究小组 ${ }^{[33]}$ 首次 
将异靛青这一染料分子应用于聚合物场效应晶体管领 域, 设计合成了一系列基于异靛青为母核的聚合物材 料, 其中, IIDDT (9a) (图 4) (表 1, 化合物 9a) 的空穴迁 移率为 $0.79 \mathrm{~cm}^{2} \cdot \mathrm{V}^{-1} \cdot \mathrm{s}^{-1}$. 在随后的工作中, 我们研究小 组 ${ }^{[34]}$ 设计合成了一系列基于异靛青为母核的 D-A 共聚 物材料, 研究了其结构和性能的关系. 通过改善调整加 工方式, 进一步将 IIDDT 的空穴迁移率提高到 $1.06 \mathrm{~cm}^{2}$ $\mathrm{V}^{-1} \cdot \mathrm{s}^{-1}$. Bao 等 ${ }^{[35]}$ 将烷基侧链更换成新型的硅氧烷 PII2T-Si (9b) (图 4) (表 1, 化合物 9b), 其空穴迁移率增 加到 $2.5 \mathrm{~cm}^{2} \cdot \mathrm{V}^{-1} \cdot \mathrm{s}^{-1}$, 他们将迁移率的提高归因于聚合 物主链间 $\pi-\pi$ 堆积距离的变小. 传统意义上来说, 烷基 链的作用主要是改善材料的溶解度, 但是我们 ${ }^{[36]}$ 的研 究表明, 烷基链的设计对聚合物迁移率具有很大的影 响. 通过对烷基链分叉位置的研究, 发现随着分叉位置 远离聚合物的主干, $\pi-\pi$ 堆积距离会逐渐变小, 而当距离 为三个亚甲基时, IIDDT-C3 (9c) (图 4) (表 1, 化合物 9c) 显示了最大迁移率 $3.62 \mathrm{~cm}^{2} \cdot \mathrm{V}^{-1} \cdot \mathrm{s}^{-1}$. 这一研究揭示了 烷基链对共轭材料性能的影响, 对聚合物材料的设计具 有十分重要的意义.

苯并二噻二唑是另一个十分具有特色的强吸电子 基团. Wudl 等 ${ }^{[37]}$ 采用联二噻吩作为给体, 苯并二噻二唑 为受体, 设计合成了 D-A 共聚物 PBBTQT (10) (图 4) (表 1 , 化合物 10), 在薄膜下采取 face-on 的排列模式, 由 X 射线衍射可知, $260{ }^{\circ} \mathrm{C}$ 退火后 $\pi-\pi$ 堆积更加紧密, 其 $\pi-\pi$ 堆积距离为 $3.5 \AA$. PBBTQT 也表现出了极佳的场效应性 能, 空穴迁移率达到了 $2.5 \mathrm{~cm}^{2} \cdot \mathrm{V}^{-1} \cdot \mathrm{s}^{-1}$.

最近几年, $\mathrm{p}$ 型聚合物 OFET 的研究发展迅速, 性能 良好的 $\mathrm{p}$ 型聚合物 OFET 的空穴迁移率均超过 $1 \mathrm{~cm}^{2}$. $\mathrm{V}^{-1} \cdot \mathrm{s}^{-1}$, 其中最高的空穴迁移率已经达到 $12 \mathrm{~cm}^{2} \cdot \mathrm{V}^{-1}$. $\mathrm{s}^{-1}$. 但是, $\mathrm{p}$ 型聚合物 OFET 还无法实现商业化, 这是主 要是由于: 分子结构与性能之间的关系还有待探索, 还 没有行之有效的设计原则; 聚合物薄膜的有序性较差, 影响场效应迁移率; 不同烷基链对于器件性能的影响也 没有完全解决; 另外, 器件的稳定性还需要提高. 这些 都是该领域亟待研究解决的核心问题, 等待着有机材料 化学家们的不断探索.

\section{4 双极传输型共轭高分子材料}

双极传输型晶体管具有降低能量功耗, 改善噪声和 操作稳定等优点 ${ }^{[38,39]}$. 目前, 实现双极传输型晶体管的 方法有两种: 层状复合和材料共混. 层状复合是早期制 备双极传输型器件的方法, 通过将 $\mathrm{p}$ 型和 $\mathrm{n}$ 型材料依次 沉积于同一个器件上, 获得双极传输型器件. 但是, 层 状复合的操作过程相对复杂, 对待溶剂的要求也比较苛 刻. 材料共混是通过真空蒸镀成膜或者溶液一次性成膜
得到双极传输型器件的方法, 它的操作十分简便, 溶剂 的选择也较为简单. 但是, 相对于这两种方法, 研究者 更希望使用具有 $\mathrm{n}$ 型和 $\mathrm{p}$ 型特点的单一组分半导体材料 来制备双极传输型晶体管. 对于双极传输型聚合物材料 来说, 电子和空穴同时为主要载流子. 而理想的聚合物 双极传输型材料, 其 HOMO 能级或 LUMO 能级与金属 电极的费米能级之间的势垒小于 $1 \mathrm{eV}$, 这样既有利于空 穴注入, 又有利于电子注入 ${ }^{[40]}$ (图 4).

近年来，一系列基于缺电子体系噻吩并吡咯二酮 (DPP)的共聚物被报道具有窄带隙双极传输型场效应性 能. 2009 年, Janssen 等 ${ }^{[41]}$ 报道了以噻吩取代的 DPP 为受 体, 噻吩为给体的 D-A 共聚物 PDPPT3-HD (11a)(图 5)(表 1, 化合物 11a). 当引入 2-已基癸烷的烷基链时, 其空穴迁移率为 $0.3 \mathrm{~cm}^{2} \cdot \mathrm{V}^{-1} \cdot \mathrm{s}^{-1}$, 电子迁移率为 0.12 $\mathrm{cm}^{2} \cdot \mathrm{V}^{-1} \cdot \mathrm{s}^{-1}$. 研究者发现, 当相对分子质量最大(54 $\mathrm{kg} / \mathrm{mol}$ ) 时得到最高电子迁移率, 而当相对分子质量较 低(10 kg/mol)时得到最高的空穴迁移率. 2012 年, Cho 等 ${ }^{[22]}$ 在 PDPP3T-OD (11b)(图 5)上引入了 2-辛基十二烷 的烷基链, 其器件的空穴迁移率为 $1.57 \mathrm{~cm}^{2} \cdot \mathrm{V}^{-1} \cdot \mathrm{s}^{-1}$, 电 子迁移率为 $0.18 \mathrm{~cm}^{2} \cdot \mathrm{V}^{-1} \cdot \mathrm{s}^{-1}$. 2011 年, Jenekhe 等 ${ }^{[43]}$ 设计 合成了 HD-PPTV (12) (图 5) (表 1, 化合物 12), 聚合物 薄膜经过 $110 \sim 250{ }^{\circ} \mathrm{C}$ 热退火后, 显示了良好的双极传 输型场效应性能, 空穴迁移率为 $0.17 \mathrm{~cm}^{2} \cdot \mathrm{V}^{-1} \cdot \mathrm{s}^{-1}$, 电子 迁移率为 $0.019 \mathrm{~cm}^{2} \cdot \mathrm{V}^{-1} \cdot \mathrm{s}^{-1}$, 由 X 射线衍射可知, 其 $\pi-\pi$ 堆积距离为 $3.92 \AA .2011$ 年, Wudl 等 ${ }^{[4]}$ 报道了一系列以 噻吩取代的 DPP 为给体, 苯并二噻二唑为受体的 D-A 共聚物 PBBT6DPP (13a) (图 5)和 PBBT12DPP (13b) (图 5) (表 1, 化合物 13a 和 13b). PBBT6DPP (13) 聚合物薄膜 经过 $200{ }^{\circ} \mathrm{C}$ 退火后, 展示了良好的双极传输型场效应性 能, 空穴迁移率为 $0.83 \mathrm{~cm}^{2} \cdot \mathrm{V}^{-1} \cdot \mathrm{s}^{-1}$, 电子迁移率为 1.36 $\mathrm{cm}^{2} \cdot \mathrm{V}^{-1} \cdot \mathrm{s}^{-1}$. 当 PBBT12DPP 的噻吩环上有更长的烷基 链时, 其聚合物薄膜经过 $240{ }^{\circ} \mathrm{C}$ 热退火后, 电子迁移率 没有太大改变, 但是空穴迁移率有了很大的提高, 达到 $1.17 \mathrm{~cm}^{2} \cdot \mathrm{V}^{-1} \cdot \mathrm{s}^{-1} .2012$ 年, Chen 等 ${ }^{[45]}$ 报道了以噻吩取代 的 DPP 为受体, 硒吩为给体的 D-A 共聚物 $\operatorname{PSeDPP}(14)$ (图 5)(表 1, 化合物 14), 在经过高沸点溶剂 和热退火处理后, 其器件的空穴迁移率达到 $1.62 \mathrm{~cm}^{2}$. $\mathrm{V}^{-1} \cdot \mathrm{s}^{-1}$, 电子迁移率为 $0.14 \mathrm{~cm}^{2} \cdot \mathrm{V}^{-1} \cdot \mathrm{s}^{-1} .2012$ 年, $\mathrm{Oh}$ 等 ${ }^{[46]}$ 和 Yang 等 ${ }^{[47]}$ 受到 $\mathrm{Bao}^{[35]}$ 等工作的启发, 将硅氧烷 基链引入了以噻吩取代的 DPP 为给体, 联二硒吩为受 体的 D-A 共聚物中 PTDPPSe-Si (15) (图 5) (表 1, 化合 物 15). PTDPPSe-Si (15)聚合物薄膜展示了极高的双极 传输型场效应性能, 经过进一步修饰, 其空穴迁移率达 到 $8.84 \mathrm{~cm}^{2} \cdot \mathrm{V}^{-1} \cdot \mathrm{s}^{-1}$, 电子迁移率达到 $4.34 \mathrm{~cm}^{2} \cdot \mathrm{V}^{-1} \cdot \mathrm{s}^{-1}$. 2012 年, 我们研究小组 ${ }^{[48]}$ 引入氟原子修饰异靛青 


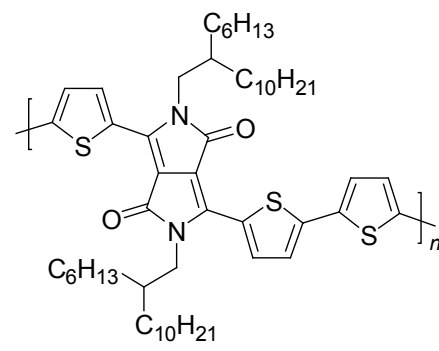

11a PDPPT3-HD

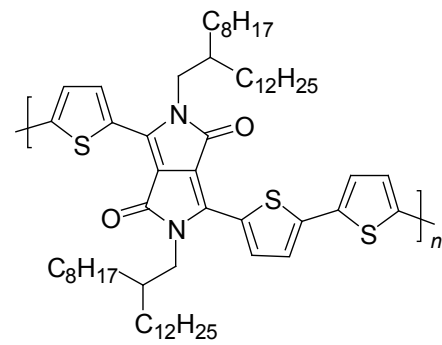

11b PDPPT3-OD

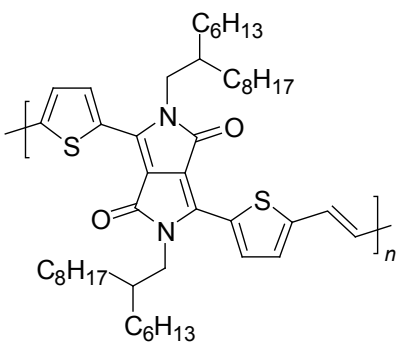

12 HD-PPTV

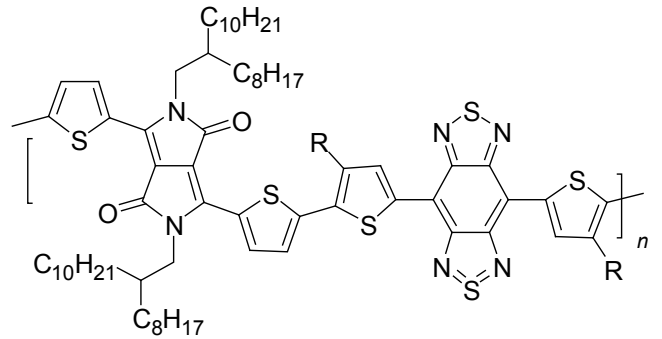

13a $\mathrm{R}=\mathrm{C}_{6} \mathrm{H}_{13}$

$13 \mathrm{~b} R=\mathrm{C}_{12} \mathrm{H}_{25}$

13 PBBT-R-DPP<smiles>CO[Si](C)(CCCCCN1C(=O)C2=C(c3ccc(-c4ccc(C)s4)s3)N(CCCCC[Si](C)(O[Si](C)(C)C)O[Si](C)(C)O[Si](C)(C)O[Si](C)(C)C)C(=O)C2=C1c1ccc(C(C)(C)C)s1)O[Si](C)(C)C</smiles>

14 PSeDPP
15 PTDPPSe-Si

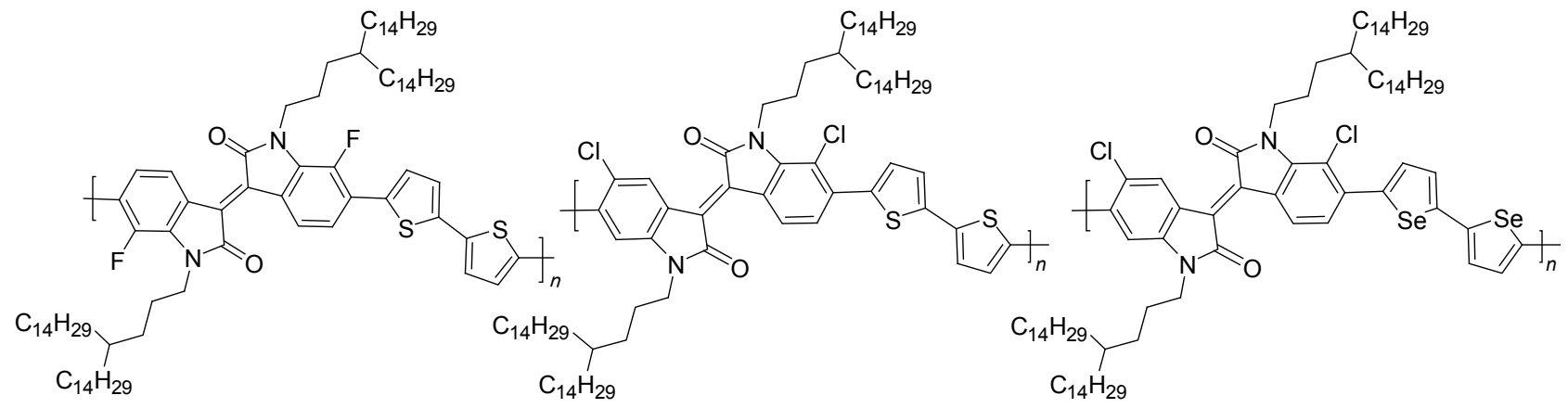

16 PFII2T

17 PCII2T

图 5 双极传输型聚合物场效应材料的分子结构式 11 18

Figure 5 Molecular structures of ambipolar materials 11 $\mathbf{1 8}$

骨架，以氟化的异靛青为受体，联二噻吩为给体，通过 Stille 偶联设计并合成 D-A 共聚物 PFII2T (16) (图 5) (表 1, 化合物 16). 引入氟原子后, 聚合物材料的 LUMO 能 级得到有效地降低, 成功实现了空气稳定的高效率空穴 和电子双极传输, 空穴迁移率达到 $1.85 \mathrm{~cm}^{2} \cdot \mathrm{V}^{-1} \cdot \mathrm{s}^{-1}$, 电 子迁移率为 $0.51 \mathrm{~cm}^{2} \cdot \mathrm{V}^{-1} \cdot \mathrm{s}^{-1} .2013$ 年, 我们研究小组 ${ }^{[49]}$ 通过高效的氯化反应简便的合成得到了氯化的异靛青, 以氯化的异靛青为受体, 联二噻吩为给体, 合成了 D-A 共聚物 PCII2T (17) (图 5) (表 1, 化合物 17). 其空穴迁移 率为 $0.54 \mathrm{~cm}^{2} \cdot \mathrm{V}^{-1} \cdot \mathrm{s}^{-1}$, 电子迁移率为 $0.48 \mathrm{~cm}^{2} \cdot \mathrm{V}^{-1} \cdot \mathrm{s}^{-1}$. 随后, 我们又以氯化的异靛青为受体, 联二硒吩为给体, 合成了 D-A 共聚物 PCII2Se (18) (图 5)(表 1, 化合物 18), 得到了平衡的双极传输型聚合物. 值得一提的是, 该聚 合物的器件是在空气中加工的, 其空穴迁移率为 0.81 $\mathrm{cm}^{2} \cdot \mathrm{V}^{-1} \cdot \mathrm{s}^{-1}$, 电子迁移率为 $0.66 \mathrm{~cm}^{2} \cdot \mathrm{V}^{-1} \cdot \mathrm{s}^{-1}$. 与氟代异
靛青聚合物相比，氯代聚合物载流子传输更加平衡. 我 们研究表明, 将氯原子引入聚合物后, 载流子迁移率和 器件的稳定性有了明显的提高.

\section{$5 \mathrm{n}$ 型共轭高分子材料}

$\mathrm{n}$ 型半导体材料是构筑逻辑互补电路的重要组成部 分. 近年来, $\mathrm{n}$ 型半导体材料获得了广泛的关注, 然而, 相对于 $\mathrm{p}$ 型半导体材料, 性能良好的 $\mathrm{n}$ 型半导体材料还 是很少. 这是因为: (1) $n$ 型半导体材料稳定性差, 容易 受到空气中氧气、水等成分的影响; (2)聚合物分子的能 级与源漏电极金属功函匹配困难，而载流子的注入和输 出需要聚合物材料的能级与源/漏电极所用金属的功函 数具有良好的匹配性. 良好的 $\mathrm{n}$ 型半导体材料要求 LUMO 能级必须和源漏电极的费米能级接近, 以利于 电子从源极注入, 从漏极输出. 但是, 通常所用的高功 
函电极(金、银等)有利于向半导体的 HOMO 能级注入空 穴, 而那些有利于向 LUMO 能级注入电子的低功函电 极(钙、铝、镁等)容易在空气中氧化, 和半导体发生反 应生成电荷复合物. 为了降低 $\mathrm{n}$ 型半导体材料的 LUMO 能级与高功函电极 (金、银等)相匹配, 通常采用在聚合 物的母核上引入强吸电子基团, 如 $\mathrm{F} 、 \mathrm{CN}$ 、羰基等.

䒬四酰亚二胺(NDI)和菲四酰亚二胺(PDI)显示了良 好的 $\mathrm{n}$ 型场效应性能, 是研究最为广泛的 $\mathrm{n}$ 型半导体材 料, 被广泛应用于小分子 $\mathrm{n}$ 型场效应晶体管当中 ${ }^{[50]}$. 荘 四酰亚二胺(NDI) 是酰胺类中比较重要的 $\mathrm{n}$ 型半导体材 料. 2007 年, $\mathrm{Zhu}$ 等 ${ }^{[51]}$ 以菲四酰亚二胺为受体, 并三噻吩
为给体，合成 D-A 共聚物 PDI-DD (19)（图 6)（表 1, 化 合物 19), 得到了一种黑色窄带隙聚合物, 显示了良好 的场效应性能, 以铝作为源/漏电极, 在真空条件下测试 其场效应晶体管电子迁移率达到了 $0.013 \mathrm{~cm}^{2} \cdot \mathrm{V}^{-1} \cdot \mathrm{s}^{-1}$. 2009 年, Facchetti 等 ${ }^{[52]}$ 设计合成了以联二噻吩为给体, 萗四酰亚二胺为受体的 D-A 共聚物 P(NDIO2D-T2) (20) (图 6)（表 1, 化合物 20), 其电子迁移率达到了 0.06 $\mathrm{cm}^{2} \cdot \mathrm{V}^{-1} \cdot \mathrm{s}^{-1}, 14$ 周后仍然可以达到 $0.01 \mathrm{~cm}^{2} \cdot \mathrm{V}^{-1} \cdot \mathrm{s}^{-1}$, 显 示了较好的稳定性. 随后, 他们采用 $\mathrm{TGBC}$ 器件结构, $\mathrm{P}(\mathrm{NDIO} 2 \mathrm{D}-\mathrm{T} 2)$ 的电子迁移率达到 $0.45 \sim 0.85 \mathrm{~cm}^{2} \cdot \mathrm{V}^{-1}$ • $\mathrm{S}^{-1[53]}$.

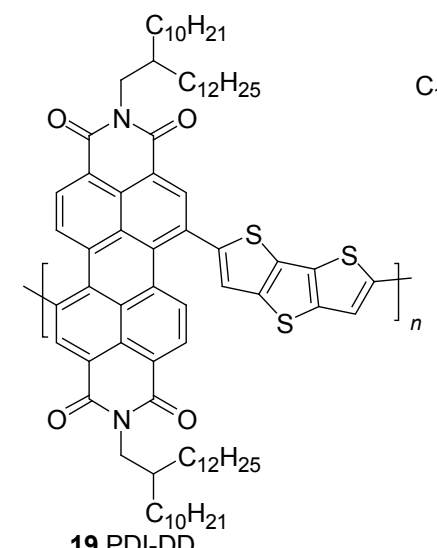

19 PDI-DD

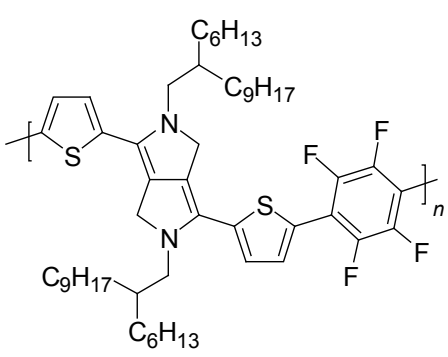

22 DPPPhF4

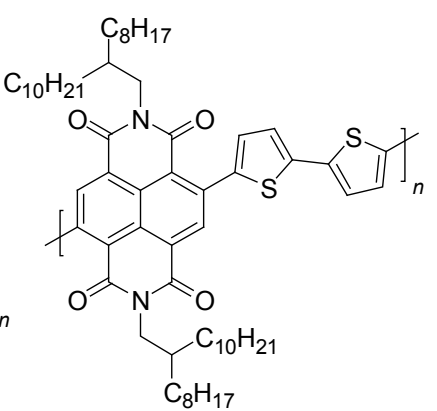

20 P(NDI2OD-T2)

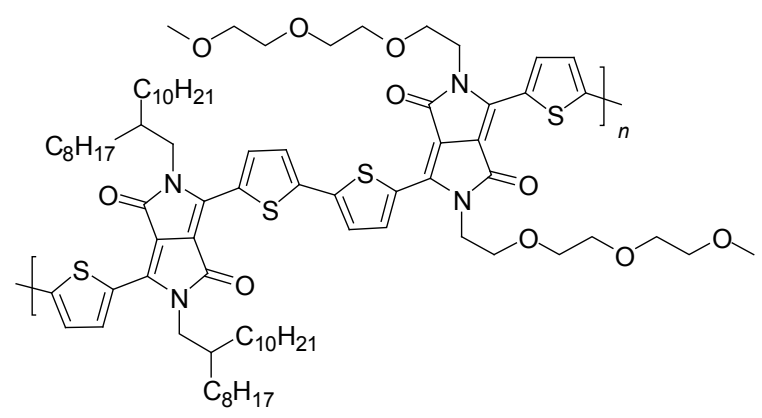

$21 \mathrm{~N}-C S 2 D P P-O D-T E G$

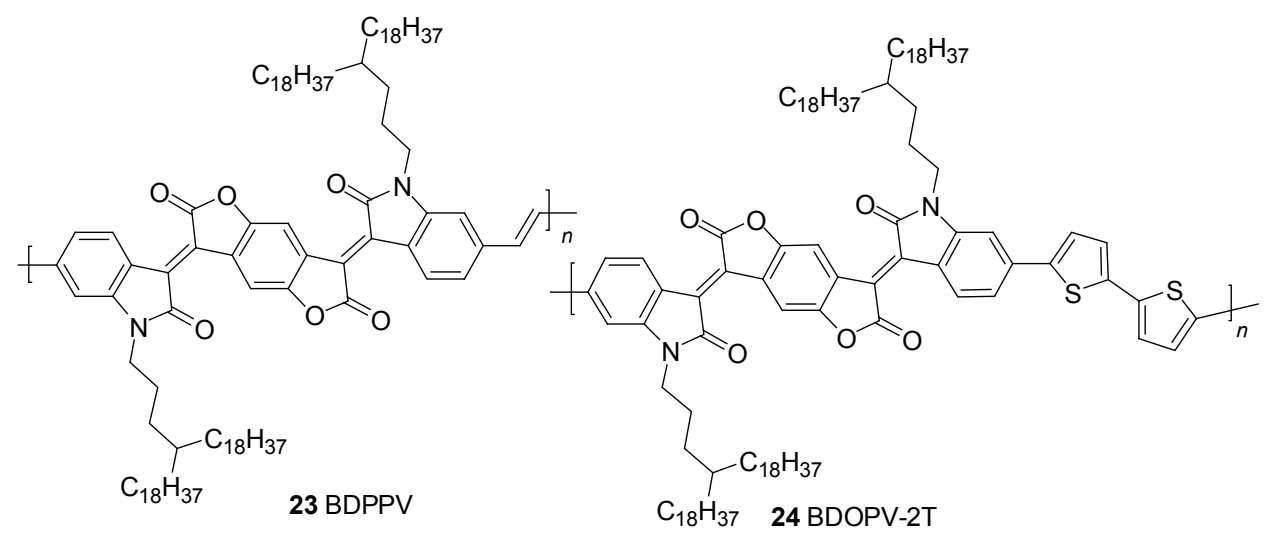

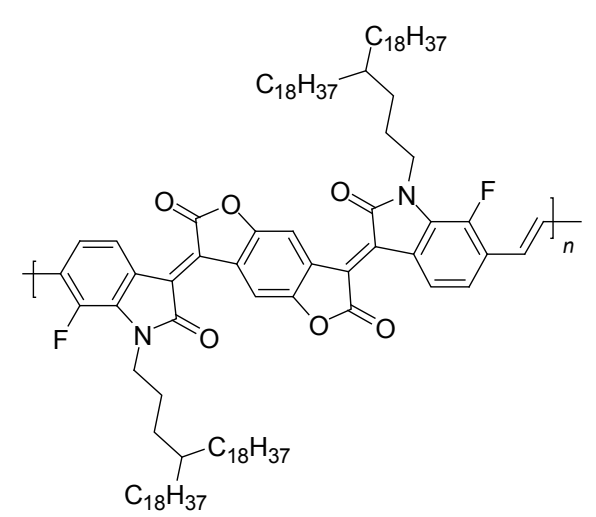

25 FBDPPV-1

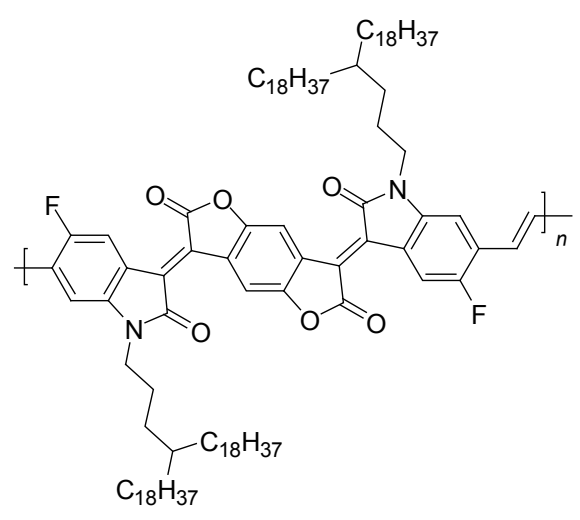

26 FBDPPV-2

图 $6 \mathrm{n}$ 型聚合物场效应材料的分子结构 19 26

Figure 6 Molecular structures of n-type host materials 19 26 
近年来，一系列基于缺电子体系并吡咯二酮(DPP) 的共聚物被报道显示了良好的 $\mathrm{n}$ 型场效应性能. 2012 年, Patil 等 ${ }^{[54]}$ 选择将两种不同烷基链的 DPP 片段进行聚合, 合成了 DPP-DPP 的 D-A 共聚物 N-CS2DPP-OD-TEG (21) (图 6)(表 1, 化合物 21). 该材料显示了良好的 n 型 场效应性能, 其电子迁移率最高可达 $3 \mathrm{~cm}^{2} \cdot \mathrm{V}^{-1} \cdot \mathrm{s}^{-1}$. 2013 年, Jo 等 ${ }^{[5]}$ 设计并合成了噻吩 DPP 与氟代苯的一系 列 D-A 共聚物. 研究发现, 对于噻吩 DPP 与四氟代苯形 成的共聚物 DPPPhF4 (22) (图 6)(表 1, 化合物 22), 四氟 代苯是比 DPP 更强的受体, 这种材料的 LUMO 能级很 低, 显示了良好的 $\mathrm{n}$ 型场效应性能, 其电子迁移率达到 了 $2.36 \mathrm{~cm}^{2} \cdot \mathrm{V}^{-1} \cdot \mathrm{s}^{-1}$.

对苯撑乙烯 $(\mathrm{PPV})$ 类聚合物是研究最早也是研究最 为广泛的一类聚合物 ${ }^{[56]}$. PPV 及其衍生物被认为是典型 的 $\mathrm{p}$-型聚合物 ${ }^{[57,58]}$, 即使使用低功函金属(如 $\mathrm{Ca}$ )和特殊 的介电层材料, PPV 也仅能够显示比较低的电子迁移率 $\left(10^{-5} \sim 10^{-4} \mathrm{~cm}^{2} \cdot \mathrm{V}^{-1} \cdot \mathrm{s}^{-1}\right)^{[59]} .2013$ 年, 我们研究小组 ${ }^{[60]}$ 设 计合成了基于苯并二呋喃二酮的 PPV, 这是一种新型的 PPV 衍生物, 简称 BDPPV (23) (图 6)(表 1, 化合物 23). 虽然该材料不是 D-A 共聚物, 但是在空气中该材料显示 了良好的 $\mathrm{n}$ 型场效应性能, 其电子迁移率高达 $1.1 \mathrm{~cm}^{2}$. $\mathrm{V}^{-1} \cdot \mathrm{s}^{-1}$, 比传统 PPV 聚合物高出 4 个数量级. 是第一个 在空气中电子迁移率超过 $1 \mathrm{~cm}^{2} \cdot \mathrm{V}^{-1} \cdot \mathrm{s}^{-1}$ 的聚合物. 从 $\mathrm{BDPPV}$ 的结构来看, 在 PPV 的双键上引入的强吸电子 基团可以降低其 LUMO 能级, 而羰基能够与苯环上的 氢形成分子内氢键，从而固定聚合物的构象使之成为一 个大的共轭平面, 有利于分子间前线轨道的重叠, 从而 促进链间的载流子传输. 选择 4-十八烷基二十二烷作为 侧链有利于增强聚合物链间的 $\pi-\pi$ 堆积, 从而提高载流 子迁移率. 器件在经过 $30 \mathrm{~d}$ 的储存后仍然表现出较好的 性能, 电子迁移率达到 $0.31 \mathrm{~cm}^{2} \cdot \mathrm{V}^{-1} \cdot \mathrm{s}^{-1}$. 随后, 我们 ${ }^{[61]}$ 又以 BDOPV 衍生物作为受体, 联二噻吩为给体设计并 合成了 D-A 共聚物 BDOPV-2T (24) (图 6) (表 1, 化合物 24). 该聚合物在空气下具有高达 $1.74 \mathrm{~cm}^{2} \cdot \mathrm{V}^{-1} \cdot \mathrm{s}^{-1}$ 的电 子迁移率. 当器件在空气中加工, 经氧气掺杂后, 表现 出了双极传输型的传输特性, 电子迁移率最高达到 1.45 $\mathrm{cm}^{2} \cdot \mathrm{V}^{-1} \cdot \mathrm{s}^{-1}$, 同时空穴迁移率可达 $0.47 \mathrm{~cm}^{2} \cdot \mathrm{V}^{-1} \cdot \mathrm{s}^{-1}$. 2014 年, 我们研究小组 ${ }^{[62]}$ 引入氟原子修饰 BDOPV 骨 架, 设计并合成了两个共轭聚合物 FBDOPV-1 和 FBDOPV-2, 它们具有较低的 LUMO 能级约为 -4.38 $\mathrm{eV}$. 基于这两个基团, 我们合成了两个 PPV 的衍生物, FBDPPV-1 (25) (图 6) (表 1, 化合物 25) 和 FBDPPV-2 (26) (图 6) (表 1, 化合物 26), 这两种聚合物适用于高性 能的 $\mathrm{n}$ 型聚合物场效应管. 氟原子的引入有效地降低两 种聚合物的 LUMO 能级, 导致了 LUMO 能级低至一
$4.30 \mathrm{eV}$. 另一方面，氟原子的引入也使薄膜分布更加有 序，使分子间作用力和聚合物骨架的锁定构象更加强 烈. 所有这些因素促成了 FBDPPV-1 的电子迁移率达到 了 $1.70 \mathrm{~cm}^{2} \cdot \mathrm{V}^{-1} \cdot \mathrm{s}^{-1}$, 并且它在空气状态下具有良好的 稳定性. 此外, 研究结果表明当聚合物的氟化位置不同, 在固态的骨干构象不同, 最终导致器件性能的不同.

目前，研究者们在 $\mathrm{n}$ 型半导体材料的设计合成上已 经有了一些进展，但是限制 $\mathrm{n}$ 型半导体材料快速发展的 几个重大问题仍然没有得到解决, 使得商业化应用难以 实现. 首先，分子结构与性能之间的关系仍不明确，而 $\mathrm{n}$ 型有机半导体材料至今没有显示出优于无机材料的性 能, 这在一定程度上影响了研究者们开发 $\mathrm{n}$ 型有机半导 体材料的动力. 其次, 高迁移率、可溶性的 $\mathrm{n}$ 型聚合物 半导体材料较少，而良好的溶解性对聚合物实现高效 率、大面积生产有着至关重要的意义, 协调聚合物材料 的溶解度和场效应性能之间的关系仍然是巨大的挑战. 最后, $\mathrm{n}$ 型半导体聚合物场效应晶体管性能和空气稳定 性还需要大幅度的提高. 因此, 只有解决上述问题, $\mathrm{n}$ 型 半导体材料的研究才能更加迅速地发展.

\section{6 总结和展望}

近两年, D-A 共聚物场效应晶体管材料的研究取得 了飞速的发展，涌现了许多新颖的高迁移率半导体材 料. 本文总结了具有不同结构的空穴传输型、双极传输 型、电子传输型的 D-A 共聚物场效应晶体管的最新进展 和应用. 与 $\mathrm{p}$ 型聚合物材料相比, 双极传输型和 $\mathrm{n}$ 型聚 合物材料的研究较少, 发展也较慢, 而 $\mathrm{n}$ 型聚合物材料 的研究是这个领域中最为困难的. 因此, 设计新的结构 以及实现高迁移率的 $\mathrm{n}$ 型聚合物材料是一个非常重要的 研究方向. 另外, 虽然已经得到了许多具有高空穴迁移 率的材料体系, 但是 D-A 共聚物中结构和性能关系的研 究还不成熟, 阻碍了该领域的发展.

为了实现高迁移率的场效应晶体管，需要 D-A 共聚 物在化学结构上符合以下几个方面的要求：合适的 HOMO/LUMO 能级、良好的共平面性，紧密有序的分子 堆积方式、良好的溶解性以及化学稳定性等. 今后, 在 设计 D-A 共聚物时，必须综合考虑这些因素，从而得到 具有高性能的场效应晶体管器件.

\section{References}

[1] Chiang, C. K.; Fincher, C. R.; Park, Y. W.; Heeger, A. J.; Shirakawa, H.; Louis, E. J.; Gau, S. C.; MacDiarmid, A. G. Phys. Rev. Lett. 1977, 39, 1098.

[2] Wu, S. K.; Wang P. F. Introduction to Organic Electronics, Chemical Industry Press, Beijing, 2010, p. 5 (in Chinese).

(吴世康, 王鹏飞, 有机电子学概论, 化学工业出版社, 北京, 2010, p. 5.) 
[3] Tsumura, A.; Koezuka, H.; Ando, T. Appl. Phys. Lett. 1986, 49, 1210.

[4] Tang, C. W. Appl. Phys. Lett. 1986, 48, 183.

[5] Tang, C. W; van Slyke, S. A. Appl. Phys. Lett. 1987, 51, 913.

[6] Crone, B.; Dodabalapur, A.; Lin, Y. Y.; Filas, R. W.; Bao, Z.; LaDuca, A.; Sarpeshkar, R.; Katz, H. E.; Li, W. Nature 2000, 403, 521.

[7] Rogers, J. A.; Bao, Z.; Baldwin, K.; Dodabalapur, A.; Crone, B.; Raju, V. R.; Kuck, V.; Katz, H.; Amundson, K.; Ewing, J.; Drzaic, P. Proc. Natl. Acad. Sci. 2001, 9, 4835.

[8] Sirringhaus, H. Science 2000, 290, 2123.

[9] Crone, B.; Dodabalapur, A.; Lin, Y. Y.; Filas, R. W.; Bao, Z.; LaDuca, A.; Sarpeshkar, R.; Katz, H. E.; Li, W. Nature 2001, 403, 521.

[10] Minemawari, H.; Yamada, T.; Matsui, H.; Tsutsumi, J.; Haas, S.; Chiba, R.; Kumai, R.; Hasegawa, T. Nature 2011, 475, 364.

[11] Li, J.; Zhao, Y.; Tan, H. S.; Guo, Y.; Di, C. A.; Yu, G.; Liu, Y.; Lin, M.; Lim, S. H.; Zhou, Y.; Su, H.; Ong, B. S. Sci. Report 2012, $2,1$.

[12] Li, Y.; Singh, S. P.; Sonar, P. Adv. Mater. $201022,4862$.

[13] Heeger, A. J. Chem. Soc. Rev. 2010, 39, 2354.

[14] Zhi, C. H.; Cheng, M., Z.; Shi, J. S.; Miao, X.; Hong, B. W.; Yong, C. Nat. Photonics 2012, 6, 591.

[15] Carsten, B.; He, F.; Son, H. J.; Xu, T.; Yu, L. Chem. Rev. 2011, 111, 1493.

[16] Cheng, Y. J.; Yang, S. H.; Hsu, C. S. Chem. Rev. 2009, 109, 5868.

[17] Yu, L.; Lee, Y.; Liang, Y. Synth. Lett. 2006, 18, 2879.

[18] Yuen, J. D.; Wudl, F. Energy Environ. Sci. 2013, 6, 392.

[19] Zhang, W.; Jeremy, S.; Roman, G.; Michael, M.; Alberto, S.; James, K.; Shahid, A.; Anthopoulos, T.; Martin, H.; McCulloch, I. J. Am. Chem. Soc. 2010, 132, 11437.

[20] Tsao, H. N.; Cho, D. M.; Park, I.; Hansen, M. R.; Mavrinskiy, A.; Yoon, D. Y.; Graf, R.; Pisula, W.; Spiess, H. W.; Müllen, K. J. Am. Chem. Soc. 2011, 133, 2605.

[21] Wang, S.; Kappl, M.; Liebewirth, I.; Müller, M.; Kirchhoff, K.; Pisula, W.; Müllen, K. Adv. Mater. 2012, 24, 417.

[22] Farnum, D. G.; Mehta, G.; Moore, G. G.; Siegal, F. P. Tetrahedron Lett. 1974, 15, 2549.

[23] Hao, Z.; Iqbal, A. Chem. Soc. Rev. 1997, 26, 203.

[24] Turbiez, M. G. R.; Janssen, R. A. J.; Wienk, M.; Kirner, H. J.; Tieke, B.; Zhu, Y. WO 2008000664, 2008 [Chem. Abstr. 2008, 148, 122502].

[25] Ha, T. J.; Sonar, P.; Dodabalapur, A. Appl. Phys. Lett. 2011, 98, 253.

[26] Sonar, P.; Singh, S. P.; Li, Y.; Ooi, Z. E.; Ha, T. J.; Wong, I.; Soh, M. S.; Dodabalapur, A. Energy Environ. Sci. 2011, 4, 2288.

[27] Zhang, X.; Richter, L. J.; De, D. M.; Kline, R. J.; Hammond, M. R.; McCulloch, I.; Heeney, M.; Ashraf, R. S.; Smith, J. N.; Anthopoulos, T. D.; Schroeder, B.; Geerts, Y. H.; Fischer, D. A.; Toney, M. F. J. Am. Chem. Soc. 2011, 133, 15073.

[28] Ha, J. S.; Kim, K. H.; Choi, D. H. J. Am. Chem. Soc. 2011, 133, 10364.

[29] Li, Y.; Sonar, P.; Singh, S. P.; Soh, M. S.; Van Meurs, M.; Tan, J. J. Am. Chem. Soc. 2011, 133, 2198.

[30] Chen, H.; Guo, Y.; Yu, G.; Zhao, Y.; Zhang, J.; Gao, D.; Liu, H.; Liu, Y. Adv. Mater. 2012, 24, 4618.

[31] Kang, I.; Yun, H. J.; Chung, D. S.; Kwon, S. K.; Kim, Y. H. J. Am. Chem. Soc. 2013, 135, 14896.

[32] Stalder, R.; Mei, J.; Reynolds, J. R. Macromolecules 2010, 43, 8348.

[33] Lei, T.; Cao, Y.; Fan, Y.; Liu, C. J.; Yuan, S. C.; Pei, J. J. Am. Chem. Soc. 2011, 133, 6099.

[34] Lei, T.; Cao, Y.; Zhou, X.; Peng, Y.; Bian, J.; Pei, J. Chem. Mater.
2012, 24, 1762.

[35] Mei, J.; Kim, D. H.; Ayzner, A. L.; Toney, M. F.; Bao, Z. J. Am. Chem. Soc. 2011, 133, 20130.

[36] Lei, T.; Dou, J. H.; Pei, J. Adv. Mater. 2012, 24, 6457.

[37] Fan, J.; Jonathan D. Y.; Cui, W.; Seifter, J.; Mohebbi, A.; Wang, M.; Zhou, H.; Heeger, A.; Wudl, F. Adv. Mater. 2012, 46, 6164.

[38] Zaumseil, J.; Sirringhaus, H. Chem. Rev. 2007, 107, 1296.

[39] Cornil, J.; Brédas, J. L.; Zaumseil, J.; Sirringhaus, H. Adv. Mater. 2007, 19, 1791.

[40] Meijer, E. J.; De, L. D. M.; Setayesh, S.; Veenendaal, E. V.; Huisman, B. H.; Blom, P. W. M.; Hummelen, J. C.; Scherf, U.; Klapwijk, T. M. Nat. Mater. 2003, 2, 678.

[41] Johan, C.; Bijleveld, A. P.; Zoombelt, S. G. J.; Mathijssen, M. M.; Wienk, M. T.; Dago, M.; Janssen, A. J. J. Am. Chem. Soc. 2009, 131, 16616.

[42] Lee, J. S.; Son, S. K.; Song, S.; Kim, H.; Lee, D. R.; Kim, K.; Ko, M. J.; Choi, D. H.; Kim, B.; Cho, J. H. Chem. Mater. 2012, 24 , 1316.

[43] Wu, P. T.; Kim, F. S.; Jenekhe, S. A. Chem. Mater. 2011, 23, 4618.

[44] Yuen, J. D.; Fan, J.; Seifter, J.; Lim, B.; Hufschmid, R.; Heeger, A. J.; Wudl, F. J. Am. Chem. Soc. 2011, 133, 20799.

[45] Lin, H. W.; Lee, W. Y.; Chen, W. C. J. Mater. Chem. 2012, 22, 2120 .

[46] Lee, J.; Han, A.; Kim, J.; Kim, Y.; Oh, J. H.; Yang, C. J. Am. Chem. Soc. 2012, 134, 20713.

[47] Lee, J.; Han, A.; Kim, J.; Yu, H.; Shin, T. J.; Yang, C.; Oh, J. H. J. Am. Chem. Soc. 2013, 135, 9540.

[48] Lei, T.; Dou, J. H.; Ma, Z. J.; Yao, C. H.; Liu, C. J.; Wang, J. Y.; Pei, J. J. Am. Chem. Soc. 2012, 134, 20025.

[49] Lei, T.; Dou, J. H.; Ma, Z. J.; Yao, C. H.; Wang, J. Y.; Pei, J. Chem. Sci. 2013, 4, 2447.

[50] Zhan, X.; Facchetti, A.; Barlow, S.; Marks, T. J.; Ratner, M. A.; Wasielewski, M. R.; Marder, S. R. Adv. Mater. 2011, 23, 268.

[51] Zhan, X.; Tan, Z.; An, B. D. Z.; Zhang, X.; Barlow, S.; Li, Y.; Zhu, D.; Kippelen, B.; Marder, S. R. J. Am. Chem. Soc. 2007, 129, 7246.

[52] Chen, Z.; Zheng, Y.; Yan, H.; Facchetti, A. J. Am. Chem. Soc. 2009, 131, 8.

[53] Yan, H.; Chen, Z.; Zheng, Y.; Newman, C.; Quinn, J. R.; Dötz, F. Kastler, M.; Facchetti, A. Nature 2009, 457, 679.

[54] Kanimozhi, C.; Yaacobi-Gross, N.; Chou, K. W.; Amassian, A.; Thomas, D.; Patil, S. J. Am. Chem. Soc. 2012, 134, 16532.

[55] Park, J. H.; Jung, E. H.; Jung, J. W.; Jo, W. H. Adv. Mater. 2013 , $25,2583$.

[56] Kraft, A.; Grimsdale, A. C.; Holmes, A. B. Angew. Chem. 1998, 37, 402.

[57] Blom, P. W. M.; Jong, M. J. M.; Munster, M. G. Phys. Rev. 1997, 55,655 .

[58] Meijer, E. J.; De, L. D. M.; Setayesh, S.; Veenendaal, E. V.; Huisman, B. H.; Blom, P. W. M.; Hummelen, J. C.; Scherf, U.; Klapwijk, T. M. Nat. Mater. 2003, 2, 678.

[59] Van, B. A. J. J. M.; Herwig, P. T.; Chlon, C. H. T.; Sweelssen, J.; Schoo, H. F. M.; Benito, E. M.; de, L. D. M.; Tanase, C.; Wildeman, J.; Blom, P. W. M. Adv. Funct. Mater. 2005, 15, 872.

[60] Lei, T.; Dou, J. H.; Cao, X. Y.; Wang, J. Y.; Pei, J. J. Am. Chem. Soc. 2013, 135, 12168.

[61] Lei, T.; Dou, J. H.; Cao, X. Y.; Wang, J. Y.; Pei, J. Adv. Mater. 2013, 23, 1 .

[62] Lei, T.; Xia, X.; Wang, J. Y.; Liu, C. J.; Pei, J. J. Am. Chem. Soc. 2014, 136, 2135.

(Zhao, X.) 